\title{
Can Capacity Markets Be Designed by Democracy?
}

\author{
Seth Blumsack \\ Penn State University and \\ Santa Fe Institute \\ sab51@psu.edu
}

\author{
Kyungjin Yoo \\ Penn State University \\ kxy151@psu.edu
}

\author{
Nicholas Johnson \\ Penn State University \\ nhj105@psu.edu
}

\begin{abstract}
Regional Transmission Organizations (RTOs) are stakeholder-driven organizations where changes to rules or protocols go through a process of stakeholder approval. Based on interviews with PJM stakeholders, we observe the perception that the process is held up by specific coalitions. We use voting data from the PJM stakeholder process and a model of participatory decision-making to assess these stakeholder perceptions, integrated with a model of PJM's capacity market to address how stakeholder-driven processes can design market constructs that promote reliability. We do observe a strong voting coalition by demand-side interests (electric distribution utilities and large directaccess customers) but not by supply-side interests. In theory, this demand-side coalition can act in a pivotal manner to prevent any rule change from going forward. In the capacity market redesign case in practice, the pivotal or swing participants are more likely a smaller segment of financial market participants, such as hedge funds and banks.
\end{abstract}

\section{Introduction}

Regional Transmission Organizations (RTOs) control the power grid serving more two-thirds of electricity customers in the U.S. and are critical organizations for ensuring reliable system operations and facilitating the integration of new technologies and market participants, including renewable power generation, energy storage and demand response. They are also supposed to be highly stakeholder-driven organizations, with rules and policies crafted through a highly participatory process. While the decisions that RTOs make have implications for industry, society and the environment, their decision processes have not been broadly studied. The environment in which rules and policies are made is important because market rules have a critical impact on the value of technology [1]. The preferences of different actors can impact the scale and scope of technology adoption [2,3], and can even affect system reliability [4].

The governance of RTOs and the behavior of RTOs as organizations has been raised a number of times in the literature (see [5-8] for examples). While questions have been raised about the outcomes of such stakeholder-driven decision processes, those processes have not, to date, been modeled in any systematic way.

RTOs are tasked with ensuring reliable system operations, but there has been tension at times between this performance goal and the design goal for RTOs of being stakeholder-driven organizations. A recent set of semi-structured interviews with stakeholders active in the PJM Interconnection identified a number of sources of this tension $[9,10]$ :

1. A change in the nature of the decisions that the stakeholder process in PJM is asked to consider, from the more general and foundational to the more specific and technical;

2. Growth in the number and nature of stakeholders has made informal coordination among stakeholders and stakeholder groups more difficult (effectively increasing transactions costs for the stakeholder process);

3. A perception among PJM staff that the increased difficulty of stakeholder coordination may threaten reliability of the electricity system - viewed within PJM as the primary mission of the RTO.

One outgrowth of these stakeholder perceptions has been to question the extent to which the rules for highly reliable system operations and planning to support reliable operations can be well-designed through a highly participatory process with so many competing interests. We investigate this question theoretically and empirically using a highly detailed data set from the PJM stakeholder process. Our work bridges some of the seminal literature from political science and political economy on the theory of voting systems [11 -16] and integrates models of the stakeholder process and market rules within PJM. We focus in particular on a series of votes taken in the PJM stakeholder process in 2011 on capacity market redesign.

Section 2 of the paper describes the PJM stakeholder process and the voting structure used in the Members Committee, the construct on which we primarily focus in this work. Section 3 describes our voting data set from PJM and uses network analysis to identify strong 
coalitions among end-user and supplier-side stakeholders in PJM. Section 4 describes the capacity market redesign alternatives, while Section 5 develops a theory of "passable proposals" that we use to parameterize capacity market designs and the payoffs to different stakeholders from those designs. In Section 6 we use our passable proposal model to closely examine the spaces of capacity market redesign proposals that could or could not pass, and also examine the potential for different groups of stakeholders to behave pivotally to keep proposed capacity market redesigns from passing. Section 7 offers some concluding thoughts and directions for future research.

\section{Voting Structure in the PJM RTO}

The stakeholder process in PJM has a complex and hierarchical structure, as outlined in Figure 1 (the full structure is available in [17]). Issues and proposed changes to rules and protocols are often initiated in one of a large number of thematic or issue-specific subcommittees. If approved by the subcommittee, proposed rule changes then move to higher-level committees, the Markets and Reliability Committee (MRC) and the Members Committee (MC). Once passed by the MRC and MC, issues are typically forwarded to the PJM Board of Managers. Our focus in this paper is on voting behavior in the $\mathrm{MC}$, for two reasons. First, detailed voting data is kept at the $\mathrm{MC}$ meetings for issues that do not clearly pass on a voice vote. Second, the MC has so-called "filing rights" in some areas of PJM governance, meaning that the $\mathrm{MC}$ can, in concept make filings directly with FERC, thus bypassing the PJM Board [18].

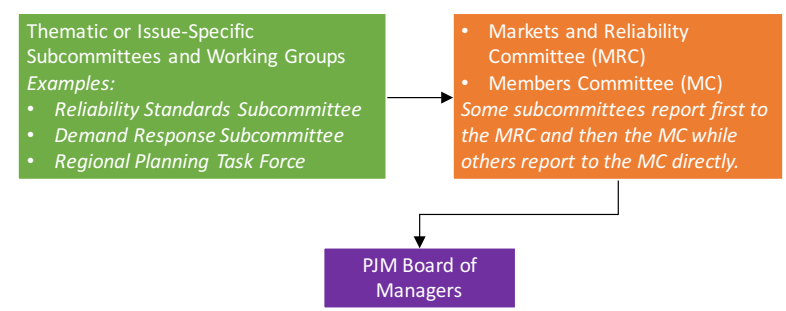

Figure 1: Structure of the PJM Stakeholder Process. Source: Adapted from [17].

Voting in the MC uses a procedure referred to by PJM as "sector-weighted voting." Stakeholder participants in the MC self-identify with one of five sectors: Generation Owners (GO), Transmission Owners (TO), Electric Distribution Utilities (EDU), End Use Customers (EUC), or Other Suppliers (OS). The number of MC voters self-identifying in each sector, along with examples of specific companies in each sector, is shown in Table 1. Stakeholder participants are permitted to switch sectors, although we did not observe this happening very often in our data set. Stakeholders have some discretion to choose the sector with which they identify, particularly if they have business units in multiple sectors (such as a generation owner with a loadserving obligation). The Other Suppliers sector is the largest and most diverse, consisting primarily of curtailment service providers, financial institutions, marketers and traders, and municipal/co-op utilities.

Table 1: Composition of MC Voters. Source: PJM [19]

\begin{tabular}{|l|l|l|}
\hline Sector & $\begin{array}{l}\text { Number of } \\
\text { Firms (\%) }\end{array}$ & Example Firms \\
\hline $\begin{array}{l}\text { End Use } \\
\text { Customers }\end{array}$ & $6(4 \%)$ & $\begin{array}{l}\text { Air Products, Proctor \& } \\
\text { Gamble }\end{array}$ \\
\hline $\begin{array}{l}\text { Electric } \\
\text { Distributors }\end{array}$ & $14(9 \%)$ & $\begin{array}{l}\text { PEPCO, Northern } \\
\text { Virginia Electric } \\
\text { Cooperative }\end{array}$ \\
\hline $\begin{array}{l}\text { Generation } \\
\text { Owners }\end{array}$ & $22(15 \%)$ & Calpine, NRG \\
\hline $\begin{array}{l}\text { Transmission } \\
\text { Owners }\end{array}$ & $5(3 \%)$ & Duquesne Light, PSEG \\
\hline Other Suppliers & $105(69 \%)$ & $\begin{array}{l}\text { Direct Energy (CSP), } \\
\text { Citigroup Energy } \\
\text { (Financial), EDF Trading } \\
\text { (Marketer) }\end{array}$ \\
\hline
\end{tabular}

Each of the five sectors is given equal weight in the PJM sector-weighted voting scheme. Within a given sector, each voting member is given equal weight. For each issue, MC participants can choose to vote yes or no, or can choose to abstain. A final voting score $V$ is calculated as:

$$
\text { (1) } V=\sum_{k} \sum_{j=1}^{\left(n_{k}-a_{k}\right)} \frac{\delta_{j k}}{n_{k}-a_{k}}
$$

where $\delta_{\mathrm{jk}}$ is an indicator variable equal to one if voter $j$ from sector $k$ voted yes and zero if voter $j$ from sector $k$ voted no, $n_{k}$ is the total number of firms in sector $k$, and $a_{k}$ is the number of abstaining firms in sector $k$. A voting item passes if it attains a voting score of $V \geq$ 3.335. This is equivalent to needing a supermajority of the five sectors for a vote to pass, and means that two sectors voting together (irrespective of the number of voters in the two sectors) could effectively act as a pivotal coalition, keeping any voting item from passing.

Because of variation in the number of $\mathrm{MC}$ participants in each of the five sectors, an individual firm's vote may contribute more in one sector than in other sectors. This phenomenon, and the MC voting system overall, is illustrated by the hypothetical voting item in Table 2.

Table 2: Sector-Weighted Voting Example 
Sector-weighted voting example

\begin{tabular}{|c|c|c|c|c|c|c|}
\hline Sector & For & Against & Abstain & Total & Total - Abstain & $\%$ in favor \\
\hline Transmission Owner & 8 & 2 & 4 & 14 & 10 & 0.8 \\
\hline Generation Owner & 15 & 0 & 1 & 16 & 15 & \\
\hline Other Supplier & 10 & 10 & 5 & 25 & 20 & \\
\hline Electric Distributor & 3 & 7 & 15 & 25 & 10 & 0.3 \\
\hline End Use Customer & 12 & 2 & 0 & 14 & 14 & 0.85 \\
\hline
\end{tabular}

In this example (which is not based on any actual data and is only for illustration), there are 94 MC voters, 25 of which abstain. There are thus 10 counted voters from the TO sector, 15 voters from the GO sector, 20 voters from the OS sector, 10 voters from the ED sector and 14 voters from the EUC sector. Of the counted voters, $80 \%$ from the TO sector voted in favor ( 8 out of 10 voters that did not abstain), yielding a voting score from that sector of 0.8 . As another example, $100 \%$ of the voters in the GO sector voted in favor, yielding a voting score of 1 for that sector. When the voting scores for all five sectors are added up, the overall voting score for this hypothetical issue is $V=3.347$. Since this is higher than the threshold score of $V=3.335$, this hypothetical voting issue would have passed.

\section{The Structure of Stakeholder Voting in PJM}

Detailed voting data from the PJM MC was obtained from the PJM web site covering the period 2011 to 2015 [19]. Data prior to 2011 was not available for PJM. Voting data for the MRC and other subcommittees is not recorded in the same level of detail as voting data from the MC. Our data set consists of firm-level voting data for 26 votes cast on a wide variety of topics. We note that the MC votes on a large number of issues each year, and most pass or fail by voice vote. The 26 voting items in our data set thus represent a subset of votes taken during the time period 2011 - 2015 when an official vote count was requested by PJM or by a member of the MC. For each voting item, the data we obtained from PJM lists the name of the voting firm, how the firm voted (or if it abstained), their sectoral affiliation and primary line(s) of business (which, as discussed in Sec. 2 , may be different than their sectoral affiliation), some information on the size of their generation holdings, transmission holdings and load obligations in PJM (i.e., whether each firm identifies as a small or large generator and identifies as a net buyer or seller), and the name of the person representing that firm on each vote.

To visualize the extent of and strength of coalitions across all 26 of the voting items, we structured the voting data as a weighted network. Each firm in the network was represented as a node, with branches representing votes. Two firms voting together on an individual voting item were thus linked by a branch in our network representation. Branches were weighted by the frequency with which firms voted together across our 26 voting items.

A visualization of the voting network for firms voting against specific voting items is shown in Figure 2 (i.e., Figure 2 shows the frequency with which firms vote together in opposition to certain issues - the frequency with which they vote together in favor of certain issues or abstain would essentially be the inverse of the network shown in Figure 2). The figure also shows the results of a community identification algorithm [20,21] that we used to identify strong coalitions in our voting network. The community detection algorithm essentially performs hierarchical clustering on the network and identifies sub-groups of voters that vote together more frequently than with other sub-groups.

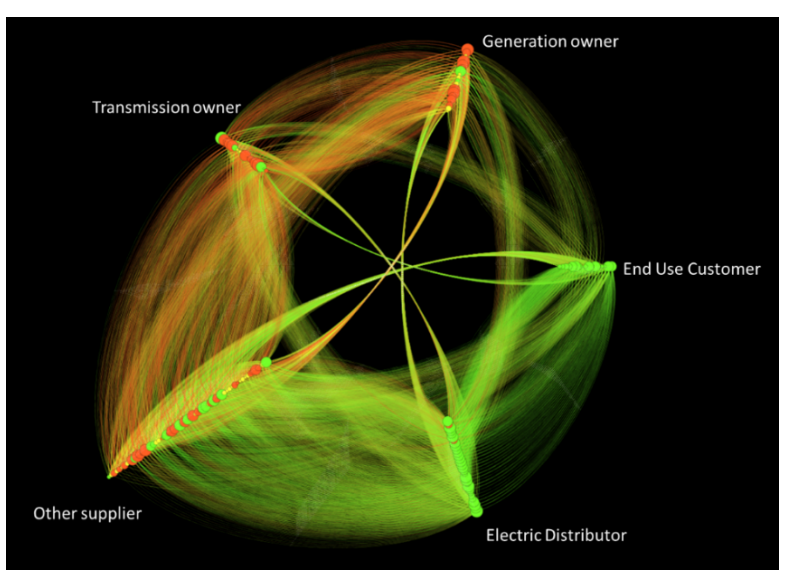

Figure 2: The Voting Network of the PJM MC.

Our clustering algorithm identified three communities in the PJM voting network, represented as green, red and yellow nodes and branches in Figure 2. As would be expected, the densities of these detected communities are individually quite high (between 0.95 and 0.99). More interesting is the composition of the detected communities. One of the communities shown in the network in Figure 2 consists primarily of firms from the ED and EUC sectors. The other two communities are split between the remaining three sectors (GO, TO and OS).

Recall from Section 1 that our semi-structured interviews revealed a perception among some participants in the PJM stakeholder process that loadside sectors (ED and EUC) form a strong coalition against the passage of some initiatives. The interviews also revealed a perception among some stakeholders that supply-side sectors (primarily GO and TO) form a strong coalition that keeps initiatives from passing. Our network analysis of the 26 voting items overall suggests that there is some evidence for a strong ED-EUC 
coalition that could stop initiatives from passing either through natural alignment or through explicit coordination. We find much less evidence from the voting network of such a strong coalition among any of the other three sectors. Strong coalitions of some voters across these three sectors to exist, as shown in Figure 2, but because they effectively divide sectors they cannot act in the same pivotal way as the ED-EUC coalition.

\section{Capacity Market Redesign in PJM}

The remainder of our analysis of the PJM stakeholder process is focused on a series of six MC votes taken on the same day in 2011. Each of these six voting items represented a proposed change to the structure of PJM's forward capacity construct, known as the Reliability Pricing Model. The proposed RPM changes followed the release of the second triennial RPM review by the Brattle Group [22]. All six proposals concerned the shape of the Variable Resource Requirements (VRR) curve, the administrative demand curve that PJM uses to determine the clearing point for the capacity market. None of the six proposals passed the MC with the required threshold voting score of 3.335 or higher, as shown in Table 3 (the details of each proposal are described in Sec. 4.1, below). The failure of the MC to pass any of the proposed RPM redesigns (including a status-quo proposal under which no changes would have been made to the VRR curve) triggered an alternative resolution process called the Enhanced Liason Committee process, under which groups of self-organized stakeholders presented RPM redesign alternatives to the PJM Board.

Table 3: Outcomes of RPM Redesign Votes

\begin{tabular}{ll|l|c|c|c|c|c|c|c}
\hline Item & Date & Voting item & TO & GO & EUC & ED & Other & \multicolumn{2}{|c}{ Voting Score } \\
\hline 04b0 & $10 / 20 / 2011$ & Status Quo & 0.083 & 0.071 & 0.083 & 0.043 & 0.056 & 0.336 & Failed \\
04b1 & $10 / 20 / 2011$ & PJM Recommendation & 0.8 & 0.833 & 0 & 0 & 0.667 & 2.3 & Failed \\
04b2 & $10 / 20 / 2011$ & Package 10 & 0.75 & 0.714 & 0 & 0 & 0.323 & 1.787 & Failed \\
04b3 & $10 / 20 / 2011$ & Package 11 & 0.167 & 0.08 & 0.909 & 0.913 & 0.235 & 2.301 & Failed \\
04b4 & $10 / 20 / 2011$ & Package 12 & 0.167 & 0.231 & 1 & 0.913 & 0.25 & 2.561 & Failed \\
04b5 & $10 / 20 / 2011$ & Package 13 & 0.333 & 0.267 & 1 & 1 & 0.513 & 3.113 & Failed \\
\hline
\end{tabular}

As can be seen from Table 3, some proposed RPM redesigns had fairly broad support among the ED and EUC sectors (such as Packages 11, 12 and 13), while others had strong opposition from the ED and EUC sectors (such as Package 10 and the PJM proposed RPM change). The status quo RPM proposal had the lowest overall voting score among any of the six RPM voting items.

The failure of the MC to pass any proposed redesign to RPM represented the first time that the stakeholder process had deadlocked in this way, and in our interviews raised concerns among both stakeholder participants and PJM staff as to the extent to which issues like RPM and resource adequacy more broadly could be handled by the current stakeholder voting structure. We take advantage of the unique number and structure of these votes, combined with our detailed voting data and a model of participatory decisionmaking to address two key questions relevant to using the stakeholder process to ensure reliability. First, we parameterize payoff functions for different types of firms in the PJM stakeholder process to generate a predictive voting model for this series of RPM votes and try to identify any change to the VRR curve that would have passed through the MC voting process. Second, we identify that while in theory the ED-EUC coalition described in Sec. 3 could (and sometimes does) behave pivotally to keep voting items from passing, in the specific case of RPM redesign the pivotal voters were more likely to be a smaller number of financial players.

\subsection{Modeling the VRR Redesign Proposals}

The six RPM votes on which we focus our analysis all concern the shape of the VRR curve, as shown in Figure 3 . The the VRR curve parameters most salient to our analysis include the Gross Cost of New Entry (CONE); the CONE net of estimated energy and ancillary services payments (referred to as "Net CONE"); and the Installed Reserve Margin (IRM), which refers to the quantity of capacity needed to meet peak demand plus the calculated capacity reserve. Under the RPM specifications at the time that the votes were taken, the VRR curve is horizontal at a level just below Gross CONE until the cleared capacity is a certain level below the IRM (point $a$ in Figure 3). The Gross CONE thus serves as the price cap in the RPM. Between point $a$ and a level of cleared capacity just above the IRM, the VRR curve slopes downward until it intersects with the Net CONE (point $b$ in Figure 3). At higher levels of cleared capacity the VRR curve continues to slope downward until the cleared capacity reaches several percentage points above IRM (point $c$ in Figure 3 ). Beyond point $c$, the VRR curve is vertical, indicating zero value to additional cleared capacity.

While the determination of the Gross and Net CONE is a complex matter involving the estimation of technology costs and market outcomes, the RPM redesign proposals considered by the $\mathrm{MC}$ in our data set ultimately amount to proposals over the level of points $a$ and $b$ on the VRR curve. No proposal considered by the $\mathrm{MC}$ at the time in question would have affected point $c$. Parameterizing the VRR curve proposals considered by the $\mathrm{MC}$ is relatively straightforward.

Figure 4 shows the six RPM proposals visually. As outlined in Table 3, one proposal represented the status quo (no change to the VRR curve); another came as a recommendation from $\mathrm{PJM}$ staff (referred to in the voting data as "package 1"); and four others were 
proposed by various PJM stakeholders. Compared to the status quo and other things being held equal, packages 11,12 , and 13 estimate future demand to be smaller than previous predictions leading the capacity price to be settled at lower level as compared to the status quo (dark blue line in Figure 4). On the other hand, packages 1 and 10 , would have set point $a$ vertically above point $b$, induce the clearing price to be at the price cap even when the supply offer is a little less than the target margin. This is about 40 percent or more than 60 percent increase in the price for package 10 and 1 respectively.

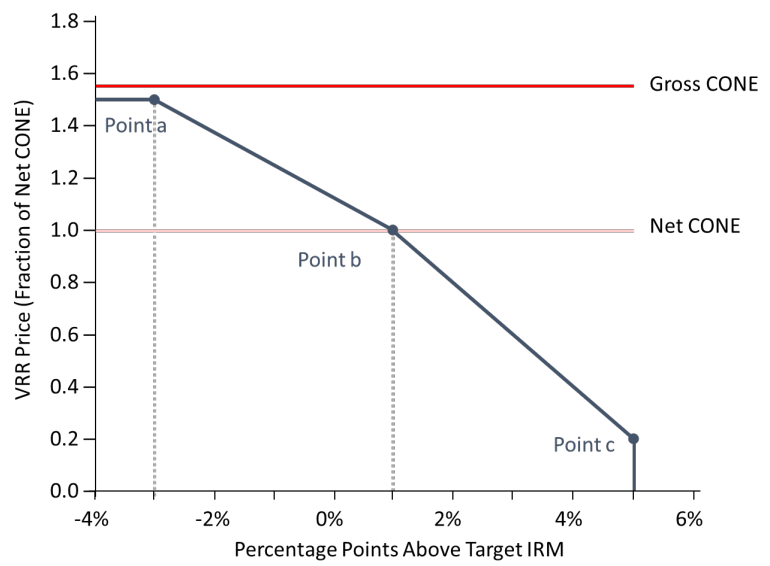

Figure 3: Example VRR Curve. Source: Author calculations based on [22]

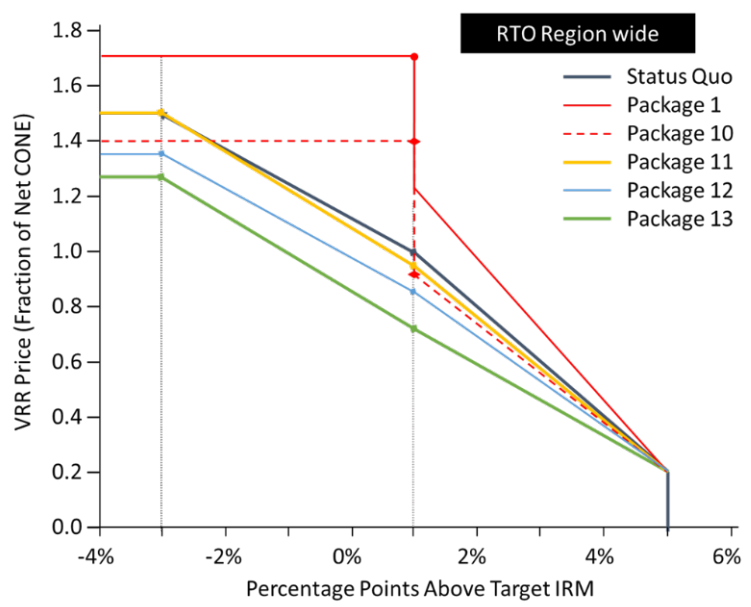

Figure 3: VRR Curves Considered by the MC. Source: Author calculations based on [22]

The set of proposed changes to the RPM considered by the MC thus consist of three proposals that would have lowered capacity prices relative to the status quo; two that would have raised capacity prices relative to the status quo; and the status quo VRR curve at the time that the proposals were voted upon.

\section{A Model of Passable Proposals}

As discussed in Sec. 3, all six of the proposed RPM redesigns failed to pass through the stakeholder process, including the status quo VRR curve. To assess the existence of any proposed capacity market redesign that would have achieved the voting score threshold for passage through the MC, we adapt the "acceptable proposal" model from the political economy literature $[15,16]$ to the structure and participant composition of the PJM Members Committee. While the acceptable proposal framework models consensus decisions, it is easily adaptable to the type of super-majority voting framework of the MC. To avoid confusion with consensus decision-making we thus refer to our framework as one to assess the existence of "passable proposals."

Suppose there are $n$ relevant policy parameters $\boldsymbol{X}=$ $\left(x_{1}, \ldots, x_{n}\right)$ (here $X$ represents the status quo); $m$ individuals and their utility functions $U^{i}=$ $U^{i}\left(x_{1}, \cdots, x_{n}\right)$ where $i=1, \cdots, m$; a proposal $\boldsymbol{y}=$ $\left(d x_{1}, \cdots, d x_{n}\right)$. If a proposal is acceptable for individual $\mathrm{i}$, then it needs to satisfy:

$$
\frac{U^{i}}{x_{1}} d x_{1}^{*}+\frac{U^{i}}{x_{2}} d x_{2}^{*}++\frac{U^{i}}{x_{n}} d x_{n}^{*}>0
$$

Equation (1) represents increase in utility of individual $i$ with the implementation of the proposal $\boldsymbol{y}^{*}$ $=\left(d x_{1} *, \ldots, d x_{n}^{*}\right)$. We assume that the payoff function of the $i$-th voter is proportional to her utility function, with $\lambda_{i}$ the constant of proportionality. Consensus in this framework is defined as the payoff function condition $\boldsymbol{A y}>\boldsymbol{0}$, where

(2)

$$
\begin{aligned}
& \frac{U^{1}}{x_{1}}
\end{aligned}
$$

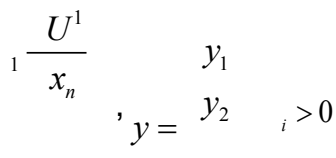

$$
\begin{aligned}
& \frac{U^{m}}{x_{1}} \quad{ }_{n} \frac{U^{m}}{x_{n}} \quad y_{n}
\end{aligned}
$$

Recall that a passable proposal in the PJM MC is one that can achieve an overall voting score of 3.335 or higher. We adapt the framework in equations (1) and (2) by defining $\boldsymbol{Z}$ as a set of firms that satisfies equation (2) and would thus vote yes on the proposal.Then a complement of $\boldsymbol{Z}, \boldsymbol{Z}^{\boldsymbol{c}}$, is a set of firms that will vote no.

Passage of a voting item in the PJM stakeholder framework needs $\sum_{i \in Z} w_{i}>3.335$ where $w_{i}$ is the 
sector-weight of a firm $i$. In other words, blockage of an issue requires $\sum_{i \in Z^{c}} w_{i}>1.665$.

If firm-level utility functions can be sufficiently parameterized, we can use the framework in equations (1) and (2) to develop a predictive model of stakeholder voting, and can apply this model to the series of RPM votes faced by the PJM Members Committee in 2011.

\subsection{Parameterizing Payoff Functions}

We use our parameterization of the capacity market proposals as specifying the shape of the VRR curve (points $a$ and $b$ in Figures 3 and 4), combined with some information on the composition of participants in the PJM Members Committee, to parameterize utility functions for different types of firms.

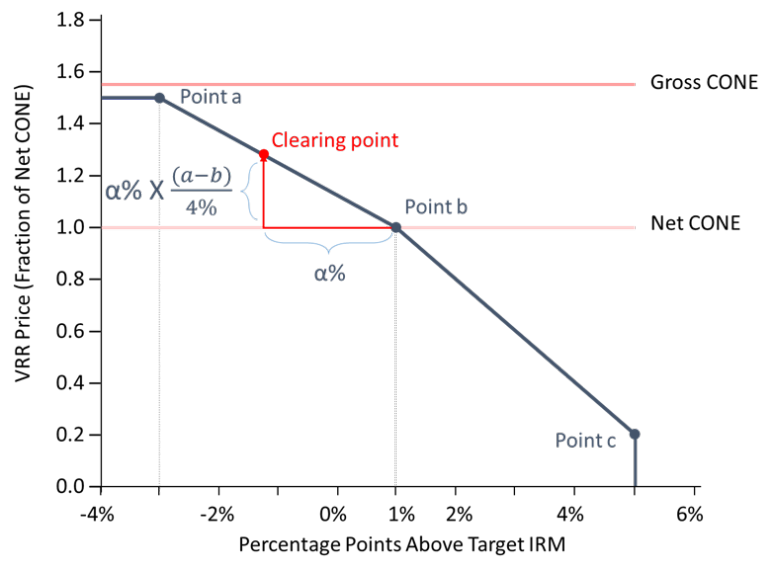

Figure 5: RPM Price Sensitivity to a Deviation of $\alpha \%$ From the IRM Target.

For the purposes of this discussion, we assume that there are five types of players in the PJM Members Committee, corresponding to the five sectoral definitions in the PJM stakeholder process (see also Sec. 2). This assumption will be relaxed somewhat below and is the topic of ongoing research. We also assume that firm-level utility can be represented by a payoff function dependent only on capacity market outcomes. In other words, we assume that payoffs from the capacity market are separable from payoffs in (for example) the energy and ancillary services markets, and firms thus vote for or against capacity market redesign proposals based solely on how each proposal would affect capacity market outcomes and not outcomes in other PJM markets. This may well be a strong assumption and is an area for future model refinement.

We assume that there are five types of players in the PJM stakeholder process, corresponding to the five stakeholder sectors: generation owner (GO), transmission owner (TO), electric distributor (ED) and end use customer (EUC) and other supplier (OS). We assume that all players within a single type are homogenous, although we will start to relax this assumption later in the paper and can relax it even further in future work. We assume that GO payoffs are directly proportional to capacity prices, while payoffs for ED and EUC are inversely proportional. Capacity payments are assumed to be made directly from ED firms and indirectly from EUC firms, so we set a share of the total capacity payment burden for ED firms as $\theta$ and the payment burden equal to $(1-\theta), \theta$ is between 0 and 1 .

The form of the payoff function for GO, ED and EUC firms will depend on whether the proposal point $y$ being considered lies to the left or right of point $b$ on the VRR curve, as shown in Figure 5. We illustrate these payoff functions assuming that the clearing quantity is below the IRM by $\alpha$ percent. In this case, the payoff functions for the GO, ED and EUC type players can be written as:

$$
\begin{gathered}
\pi_{G O}\left(P_{W}, Q, C\right)=\left(P_{W}-C\right) \times Q_{W}+\left\{b+\alpha \times \frac{(a-b)}{4}\right\} \times Q_{R} \\
\pi_{E D}\left(P_{\text {NetRet }}, Q\right)=P_{\text {NetRet }} \times Q_{W}-\theta\left\{b+\alpha \times \frac{(a-b)}{4}\right\} \times Q_{R} \\
\pi_{E U C}\left(P_{\text {Ret }}, Q\right)=-P_{\text {Ret }} \times Q_{W}-(1 \\
-\theta)\left\{b+\alpha \times \frac{(a-b)}{4}\right\} \times Q_{R}
\end{gathered}
$$

where $P_{W}$ is the clearing price in the wholesale market, $C$ is the marginal cost of generation, $P_{\text {NetRet }}$ is the retail rate net of transmission charges and wholesale costs, $P_{R}$ is the gross retail rate, $Q$ is the wholesale clearing quantity, $Q_{W}$ is the cleared quantity in the wholesale market, $Q_{R}$ is the cleared quantity in the capacity market and $\theta$ is the ED's share of capacity costs as described above.

Modeling the payoff function for the TO sector is somewhat more complex, since a firm owning no assets other than transmission would neither benefit nor be harmed by higher or lower capacity prices. We note from our data, however, that all but one of the firms in the TO sector are investor owned utilities with generation assets and load serving obligations; many voted for those capacity market proposals that would have tended to increase the capacity price. We parameterize the payoff function for the TO sector as being composed of a weighted sum of payoffs from the generation, transmission and load serving business functions: 
(4)

$\pi_{T O}\left(P_{w}, P_{T}, P_{\text {NetRet }} C, Q_{W}, Q_{R}\right)=\gamma_{1}\left\{\left(P_{w}-C\right) \times\right.$ $\left.Q+\left(b+\alpha \times \frac{a-b}{4}\right) \times Q_{R}\right\}+\gamma_{2}\left(P_{T} \times Q\right)+\gamma_{3}\left\{\left(P_{\text {NetRet }} \times\right.\right.$ Q) $\left.-\theta \times\left(b+\alpha \times \frac{a-b}{4}\right) \times Q_{R}\right\}$

In equation (4), $\gamma_{i}$ is a share of asset $i$, where $0<$ $\gamma_{i}<1, i=1,2,3$ (generation, transmission, load server respectively) and $\gamma_{1}+\gamma_{2}+\gamma_{3}=1 . \quad P_{T}$ represents the transmission fee.

From (4), we calculate marginal payoffs for this sector as:

(5)

$$
\begin{gathered}
\frac{\partial \pi}{\partial a}=\frac{\alpha}{4} Q_{R}\left(\gamma_{1}-\theta \gamma_{3}\right) \\
\frac{\partial \pi}{\partial b}=\left(1-\frac{\alpha}{4}\right) Q_{R}\left(\gamma_{1}-\theta \gamma_{3}\right)
\end{gathered}
$$

From (5), note in particular that payoffs for the TO sector do not depend on the share of its business in the transmission sector $\left(\gamma_{2}\right)$. Whether payoffs increase or decrease with marginally higher values of $a$ and $b$ in (5) depends on the quantity $\gamma_{1}-\theta \gamma_{3}$. Our model thus predicts that firms in the TO sector with large quantities of generation would vote in favor of proposals that would tend to increase capacity prices.

Parameterizing the payoff functions for the OS sector is also difficult, since that sector is highly heterogeneous, consisting of curtailment service providers, marketers, municipal and cooperative utilities, other transmission-dependent utilities, and purely financial players (such as banks and hedge funds that participate in PJM primarily via virtual bidding). We observe in our data that curtailment service providers vote with generation owners in support of capacity market proposals that would, other things being equal, lead to higher capacity clearing prices. Municipal, cooperative, and transmission-dependent utilities with generation holdings also tended to vote in alignment with the GO sector. Marketers and financial players are the most difficult to model, so we will treat these participants as "undecided" or swing voters in our model.

\section{The Geometry of Capacity Market Voting}

We are now in a position to integrate the theory of passable proposals in the PJM stakeholder process (equations 1 and 2) with our parameterization of the proposed changes to the RPM and the payoff functions of different voter types (equations 3 through 5) to identify conditions under which a proposal for reform to the VRR curve would be passable. As we will see, the existence of a passable proposal depends on both the proposal characteristics (points $a$ and $b$ ) and the voting patterns of the OS sector.

Let $\mathbf{M}$ be a matrix consisting of rows of $\mathbf{A y}$ satisfying:

(6) $\boldsymbol{M y}=\left[\begin{array}{ll}m_{i, a} & m_{i, b}\end{array}\right]\left[\begin{array}{l}d a \\ d b\end{array}\right]>0$

where $m_{i, a}$ and $m_{i, b}$ represent the marginal payoff to player $i$ from a change in $a$ and $b$, respectively. Inserting equations (3) - (5) into equations (1) and (2), the conditions for acceptability are given by:

$$
\begin{aligned}
& \boldsymbol{A} \boldsymbol{y}=\left[\begin{array}{llr}
\lambda_{G O} \frac{\partial U^{G O}}{\partial a} & \lambda_{G O} \frac{\partial U^{G O}}{\partial b} \\
\lambda_{E D} \frac{\partial U^{E D}}{\partial a} & \lambda_{E D} \frac{\partial U^{E D}}{\partial b} \\
\lambda_{E U C} \frac{\partial U^{E U C}}{\partial a} & \lambda_{E C U} \frac{\partial U^{E U C}}{\partial b} \\
\lambda_{T O} \frac{\partial U^{T O}}{\partial a} & \lambda_{T O} \frac{\partial U^{T O}}{\partial b} \\
\lambda_{C S P} \frac{\partial U^{C S P}}{\partial a} & \lambda_{C S P} \frac{\partial U^{C S P}}{\partial b} \\
\lambda_{O S} \frac{\partial U^{O S}}{\partial a} & \lambda_{O S} \frac{\partial U^{O S}}{\partial b}
\end{array}\right]\left[\begin{array}{c}
d a \\
d b
\end{array}\right]
\end{aligned}
$$

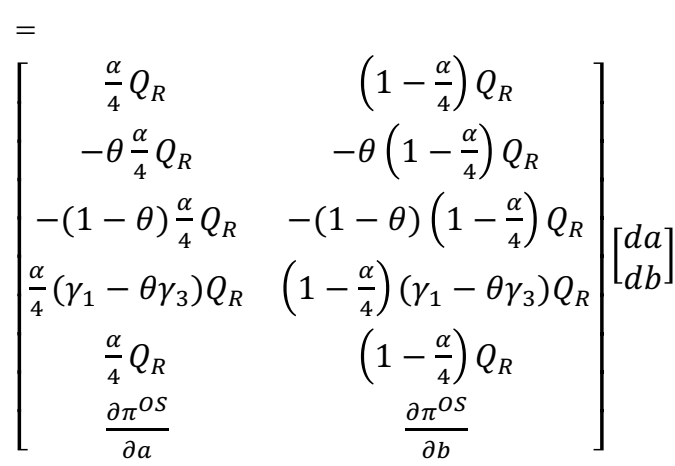

Note that in the system of equations (7), we have purposefully left the derivatives $\frac{\partial U^{O S}}{\partial a}$ and $\frac{\partial U^{O S}}{\partial b}$ ambiguous for players in the OS sector whose incentives are difficult to identify based on player characteristics or voting records.

The size of the matrix $\boldsymbol{M}$ (i.e,. the number of TO players who would find a given RPM design proposal acceptable). depends on the sign of $\gamma_{1}-\theta \gamma_{3}$. This is because TO profits differ according to the the importance of the generation versus load serving side of the business, as shown in equation (4). 
Consider the acceptability criterion for TO when the capacity market clears at a level lower than the target IRM:

(7)

$$
\left(\gamma_{1}-\theta \gamma_{3}\right)\left(\frac{\alpha}{4} Q_{R} \times d a+\left[1-\frac{\alpha}{4}\right] Q_{R} \times d b\right)
$$

If $\gamma_{1}-\theta \gamma_{3}>0$, then we have:

$$
\begin{gathered}
\left(\frac{\alpha}{4} Q_{R} \times d a+\left[1-\frac{\alpha}{4}\right] Q_{R} \times d b\right)>0 \\
\Rightarrow d b>-\frac{\alpha}{4-\alpha} \times d a
\end{gathered}
$$

and the TO firm would be predicted to vote in alignment with the GO sector. If $\gamma_{1}-\theta \gamma_{3}<0$ we would then have $d b<-\frac{\alpha}{4-\alpha} \times d a$ and the TO firm would be predicted to vote in alignment with the ED and EUC sector.

Assuming that CSPs have payoff functions equivalent to those of the GO sector, we can draw the payoff functions in the space of modifications to the capacity market as shown in Figure 6. Since the payoffs are non-overlapping, whether a given proposal passes or not would depend on the number of undecided stakeholders that could be convinced to vote for or against it.

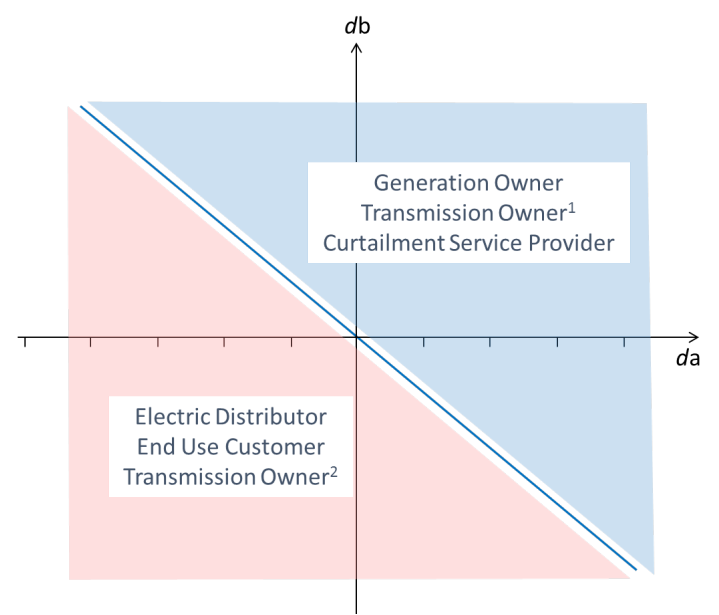

Figure 6: The space of possible modifications to the capacity market.

By way of illustration, suppose that a proposal is represented by one of the arrows in Figure 7. It would thus be acceptable to the GO sector, CSPs and those TOs for which $\gamma_{1}-\theta \gamma_{3}>0$. We would then have:
(9)

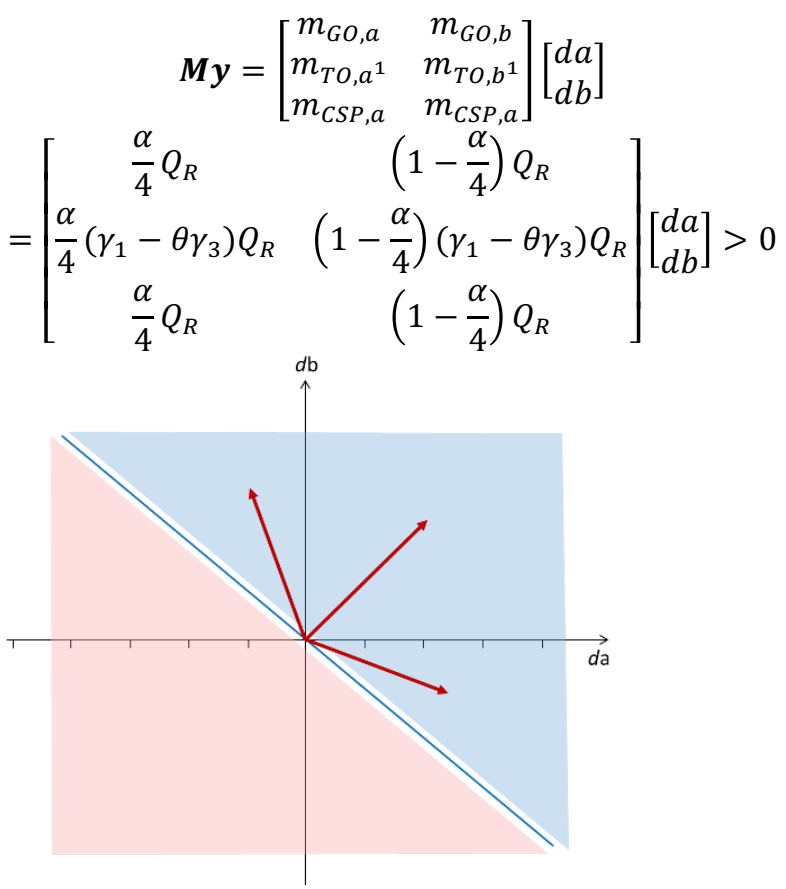

Figure 7: Representation of proposals in stakeholder preference space.

Whether such a proposal would pass can be evaluated by looking at the sector-weighted voting share contributed by those stakeholders that would vote for it, those that would vote against, and those that are undecided and act as swing voters.

\subsection{Application from the RPM Voting Data Set}

The geometry of voting in the case of the RPM redesign proposals and the influence of "undecided" voters can be illustrated with two examples, taken from a proposal that would have increased the capacity clearing price, other things being equal, relative to the status quo. We will evaluate this proposal using the coalition model developed in this paper to predict how firms would vote, and then using the actual voting data.

In the language of Section 5, Figure 7 suggests that we would have $\boldsymbol{Z}=\left\{G O, T O^{1}, C S P\right\}, \boldsymbol{Z}^{\boldsymbol{c}}=$ $\left\{E D, E U C, T O^{2}\right\}, \mathbf{U}^{c}=\{O S\}$ (where OS excludes the CSPs and other firms with alignment with other sectors). Using the mechanism of sector-weighted voting employed in PJM, if voters in coalition $\mathbf{Z}$ vote in favor, the voters in coalition $\mathbf{Z}^{\mathbf{c}}$ vote in opposition, we would have $\quad \sum_{i \in Z} w_{i}=1.95, \sum_{i \in Z^{c}} w_{i}=2.25$, and $w_{O S}=$ 0.8. Thus, under PJM rules, our model predicts that either coalition $\mathbf{Z}$ or $\mathbf{Z}^{\mathbf{c}}$ could have blocked the proposal from passing. Since $\mathbf{Z}^{\mathbf{c}}$ consists primarily of the ED and EUC sector, this illustration suggests that a demand-side 
coalition could theoretically block any capacity market redesign proposal that would tend to increase the capacity clearing price.

In practice, however, we observe some deviations from our voting model. Some firms in the GO sector, for example, voted against capacity market proposals that would have tended to raise capacity clearing prices relative to the status quo. Some firms in the ED and EUC sectors voted for such proposals. Based on observed voting behavior, we have $\sum_{i \in \boldsymbol{Z}} w_{i}=1.658, \sum_{i \in Z^{c}} w_{i}=$ 2.52 , and $w_{O S}=0.822$.

The role of the undecided or swing voters from the OS sector is more clear in practice than in theory. Under PJM sector-weighted voting rules, coalition $\mathbf{Z}^{\mathbf{c}}$ needed only two votes from the undecided OS sector in order to ensure failure of the capacity market proposal (recall that a coalition can prevent a proposal from passing with a total voting score of 1.655 or greater). Recall that the undecided OS sector consists primarily of firms that do not take physical positions in the market (such as marketers, banks and hedge funds) and as such do not have any apparent stake in capacity market outcomes. It is not immediately clear why these firms in the OS sector are voting for or against the various capacity market redesign proposals. These firms may have other business units that do have a stake in physical market outcomes (for example, banks may be involved in project finance) or they may be engaged in coalitionbuilding that spans votes on multiple issue types [23].

\section{Conclusions and Future Work}

Regional Transmission Organizations are critical organizations for ensuring reliability and facilitating the integration of new technology in the U.S. electric power grid. The rules and protocols that influence the value of new technology and the cost of achieving reliable operations are not created in a vacuum, but are rather the result of a stakeholder-driven process that we argue can be modeled in a systematic way. We have illustrated two potential ways in which formal models of the stakeholder process could be utilized - to verify stakeholder perceptions of power in the stakeholder process, and to address the increasingly relevant question of whether electricity market rules can be successfully designed directly by the players in those markets. We draw three conclusions from this work.

First, we do find evidence in a broad set of voting data that end-use interests (the ED and EUC sectors) do form a strong coalition that can and does act to strike down proposals before the MC. This is consistent with the perceptions viewed by some stakeholders in our semi-structured interviews. We do not find evidence of a similarly strong coalition among supplier-side interests.
Second, our application of the passable-proposal model to the capacity market shows how in theory either a coalition of end-use sectors and some transmission owners, or a coalition of supply-side participants and other transmission owners could keep any capacity market redesign proposal from passing. This theoretical finding suggests that there may be limits to the degree to which organizations like RTOs can create mechanisms for heterogeneous stakeholders with opposing interests to develop passable market rules and protocols.

Third, the reality of voting in the capacity market redesign case suggests that the formation of such "pivotal coalitions" is more complex than our model of passable proposals would suggest. While in theory clean-cut coalitions of end-use or supply-side interest could act to keep capacity market redesign proposals from passing, in practice the formation of these coalitions depends on convincing a small number of swing players to vote in alignment with the coalition. These swing players, primarily marketers and financial firms, represent the pivotal voters in the capacity market redesign case. Identifying the types of pivotal voters or coalitions for other voting issues is a topic of ongoing research.

Our analysis suggests that market-driven constructs to ensure resource adequacy may not be amenable to design by the type of stakeholder-driven processes currently in place in PJM. Given the controversy over capacity market constructs and the financial stakes, this is perhaps not surprising.

The history of difficulty in making changes to the RPM design was the justification for the use of an alternative mechanism for the construction of PJM's recent capacity performance rules [24]. Under this alternative mechanism, known as the Enhanced Liason Committee (ELC), stakeholders participate in the policy formation process but not through voting. Coalitions of stakeholders are self-organized, rather than stakeholders being associated with one of the five industry sectors described in this paper (a choice which, as discussed, affects the voting power of each stakeholder). In the capacity performance mechanism design process, nearly a dozen coalitions emerged from the PJM stakeholder group. Each coalition produced a position paper, and the PJM Board ultimately made the decision on which proposal to support and file with the FERC.

From an organizational theory standpoint, the ELC process actually has some advantages to the more structured stakeholder voting procedure. Because of its mission to make decisions in a stakeholder-driven way, RTOs share many commonalities with "boundary organizations," described in the organizational theory literature [25] as those who need to reconcile diverse and conflicting interests. The ELC represents a step 
towards PJM acting as this type of organization by disrupting the type of power balance observed in our analysis and allowing coalitions to emerge rather than be defined by sectoral affiliation. While the outcome is not guaranteed to be one that is universally accepted, PJM should consider expanding its use by identifying issue types where the traditional stakeholder process is likely to deadlock and invoking the ELC (or something like it) in its place.

As a mechanism for identifying these types of controversial issues, the approach used in this paper has some potential but additional work is needed to identify drivers behind deviations from coalition voting, which we observed in our capacity market analysis. Additional future work involves comparisons between PJM and other Regional Transmission Organizations. Since we find some evidence suggestive of dynamic interplay between stakeholders, particularly between traditional players in the physical market and what appear to be purely or primarily financial players, another area of future work involves using a more dynamic or gametheoretic framework to model the repeated interactions of participants in the PJM stakeholder process.

\section{Acknowledgements}

The authors acknowledge support from the U.S. National Science Foundation under award SES1261867. We also thank participants at the CRRI Eastern Workshop on Regulation and Competition for helpful comments and suggestions.

\section{References}

[1] Paine, N., Homans, F. R., Pollak, M., Bielicki, J. M., \& Wilson, E. J. (2014). Why market rules matter: Optimizing pumped hydroelectric storage when compensation rules differ. Energy Economics, 46, 10-19.

[2] Fischlein, M., Feldpausch-Parker, A. M., Peterson, T. R., Stephens, J. C., \& Wilson, E. J. (2014). Which way does the wind blow? Analysing the state context for renewable energy deployment in the United States. Environmental Policy and Governance, 24(3), 169-187.

[3] Wilson, E. J., \& Stephens, J. C. (2009). Wind deployment in the United States: states, resources, policy, and discourse. Environmental science \& technology, 43(24), $9063-$ 9070 .

[4] Carreras, B. A., Newman, D. E., Dobson, I., \& Zeidenberg, M. (2009, January). A simple model for the reliability of an infrastructure system controlled by agents. In System Sciences, 2009. HICSS'09. 42nd Hawaii International Conference on (pp. 1-10). IEEE.

[5] Dworkin, M. H., and Goldwasser, R. A. (2007). "Ensuring consideration of the public interest in the governance and accountability of regional transmission organizations.” Energy Law Journal 28(2): 543-601.
[6] Joskow, P. L. (1997). "Restructuring, competition and regulatory reform in the US electricity sector." The Journal of Economic Perspectives 11(3): 119-138.

[7] Roe, E., Schulman, P., 2008. High Reliability Management. Stanford University Press, Stanford

[8] Schulman, P., \& Roe, E. (2016). Reliability and Risk: The Challenge of Managing Interconnected Infrastructures. Stanford University Press.

[9] Johnson, N. S. Blumsack, E. Wilson, B. Stafford. (2015 September). Of Social Structures and Infrastructure: Voting Networks in Regional Transmission Organizations. Energy Policy Research Conference, Denver CO.

[10] Johnson, N. S. Blumsack, S. Lenhart, E. Wilson, B. Stafford. (2016, May.) Voting in the PJM Regional Transmission Organization. Industry Studies Association Annual Meeting, Minneapolis MN.

[11] Arrow, K. J. (1950). "A difficulty in the concept of social welfare." The journal of political economy: 328-346.

[12] Black, D., et al. (1958). The theory of committees and elections, Springer.

[13] Downs, A. (1957). "An economic theory of political action in a democracy." The journal of political economy: 135150.

[14] Rubinstein, A. (1980). "Stability of decision systems under majority rule." Journal of Economic Theory 23(2): 150159.

[15] Plott, C. R. (1967). "A method for finding "acceptable proposals" in group decision processes." Public Choice 2(1): 45-59.

[16] Plott, C. R. (1967). "A notion of equilibrium and its possibility under majority rule." The American Economic Review 57(4): 787-806.

[17] PJM, 2015. PJM Manual 34: PJM Stakeholder Process, Operator Manuals.

[18] PJM, 2015. Federal Power Act Sections 205 and 206 [WWW Document]. URL http://www.pjm.com/ /media/about-pjm/newsroom/factsheets/federal-power-act-sections-205-and-206.ashx

[19] PJM, 2015. Rosters of the Members Committee, available at http://www.pjm.com/committees-andgroups/committees/mc.aspx.

[20] Newman, M. E. (2004). Fast algorithm for detecting community structure in networks. Physical review E, 69(6), 066133.

[21] Porter, M., J. Onnela, P. Mucha (2008). Communities in Networks. Notices of the AMS 56(9), 1082-1096.

[22] Pfeiffenberger, J., K. Spees, S. Newell (2011). Second Performance Assessment of PJM's Reliability Pricing Model, available at http://www.brattle.com/system/news/pdf2s/000/000/196/origi nal/Second_Performance_Assessment_of_PJM's_Reliability Pricing_Model_Pfeifenberger_et_al_Aug_26_2011.pdf?137 $\overline{7} 791290$

[23] Buchanan, J. M., \& Tullock, G. (1962). The calculus of consent (Vol. 3). Ann Arbor: University of Michigan Press.

[24] PJM Enhanced Liason Committee, http://www.pjm.com/committees-and-

groups/committees/elc.aspx.

[25] Guston, D.H., 2001. Boundary organizations in environmental policy and science: an introduction. Sci. Technol. Hum. Values 26, 399-408. 